\title{
Candida albicans keratitis modified by steroid application
}

\author{
Kaoru Araki-Sasaki' \\ Hiroko Sonoyama' \\ Tsutomu Kawasaki' \\ Nariyasu Kazama' \\ Hidenao Ideta' \\ Yoshitsugu Inoue ${ }^{2}$
}

'Ideta Eye Hospital, Kumamoto City, Kumamoto, Japan; ${ }^{2}$ Department of Ophthalmology, Tottori University, Koyama-Minami, Tottori, Japan
Correspondence: Kaoru Araki-Sasaki Ideta Eye Hospital, 39, Nishi-tojincyo, Kumamoto City, 8600027, Kumamoto, Japan

Tel +8I 963255222

Fax +81963115512

Email sasakis@sa2.so-net.ne.jp

\begin{abstract}
The paper reports on Candida albicans ocular infection modified by steroid eye drops. A 74-year-old male complained of conjunctival injection and pain in his right eye three months after pterygium and cataract surgery. After treatment with antibiotics and steroid eye drops for three days, he was referred to our hospital. Clear localized corneal endothelial plaque with injection of ciliary body was observed. No erosion of the corneal epithelium, or infiltration of stromal edema was observed, suggesting that the pathological organism derived from the intracameral region. Because ocular infection was suspected, steroid eye drops were stopped, which led immediately to typical infectious keratitis in the pathological region, with epithelial erosion, fluffy abscess, stromal infiltration, and edema. For diagnostic purposes, the plaque was surgically removed with forceps and the anterior chamber was irrigated with antibiotics. The smear and culture examination from the plaque revealed C. albicans surrounded by neutrophils. However, aqueous fluid and fibrous tissue after gonio procedure contained no mycotic organisms. Topical fluconazole, micafungin, and pimaricin with oral itraconazole $(150 \mathrm{mg} / \mathrm{day})$ were effective. Special attention is needed when prescribing steroid eye drops to treat corneal disease especially postoperatively. Diagnosing infectious keratitis is sometimes difficult because of modification by some factors, such as postoperative conditions, scarring, and drug-induced masking. Here, we report on mycotic keratitis modified by postoperative steroid administration.
\end{abstract}

Keywords: Candida albicans, cataract surgery, steroid, mycotic keratitis

\section{Case report}

A 74-year-old man underwent pterygium excision and cataract surgery at the same day in his right eye on June 2, 2006. At the time of cataract surgery (phacoemulsification), the posterior capsule was ruptured and anterior vitrectomy was performed before intraocular lens implant. $0.5 \%$ levofloxacin and $0.1 \%$ fluorometholone eye drops were applied for one month in the usual way. His corrected visual acuity was 0.5. On September 29, 2006, he suddenly complained of redness with a foreign body sensation in his right eye, and visited a general practitioner (GP), who prescribed $0.5 \%$ levofloxacin and $0.1 \%$ fluorometholone eye drops. Three days of treatment resolved the foreign body sensation, but the cilial injection continued, and he was referred to our hospital. Slit lamp examination disclosed clear corneal endothelial plaque and fibrous tissue in the anterior chamber with no remarkable corneal focus (Figure 1). Although the stromal opacity was recognized as the scar of the pterygium excision, stromal infiltrate and edema were not observed. The corneal epithelium was not eroded as if the pathogenic organism derived from the intracameral region. The patient was treated with $0.1 \%$ betamethasone sodium phosphate and $10 \mathrm{mg}$ of oral predonisolone acetate with $0.5 \%$ levofloxacin eye drops for possible uveitis. Because no improvement was observed for a week, and infectious disease including postoperative endophthalmitis was suspected, $0.1 \%$ betamethasone sodium phosphate and $10 \mathrm{mg}$ predonisolone acetate were discontinued. Instead, $0.1 \%$ levofloxacin was applied continuously. On the day after finishing steroid therapy, the typical appearance of mycotic keratitis, such 


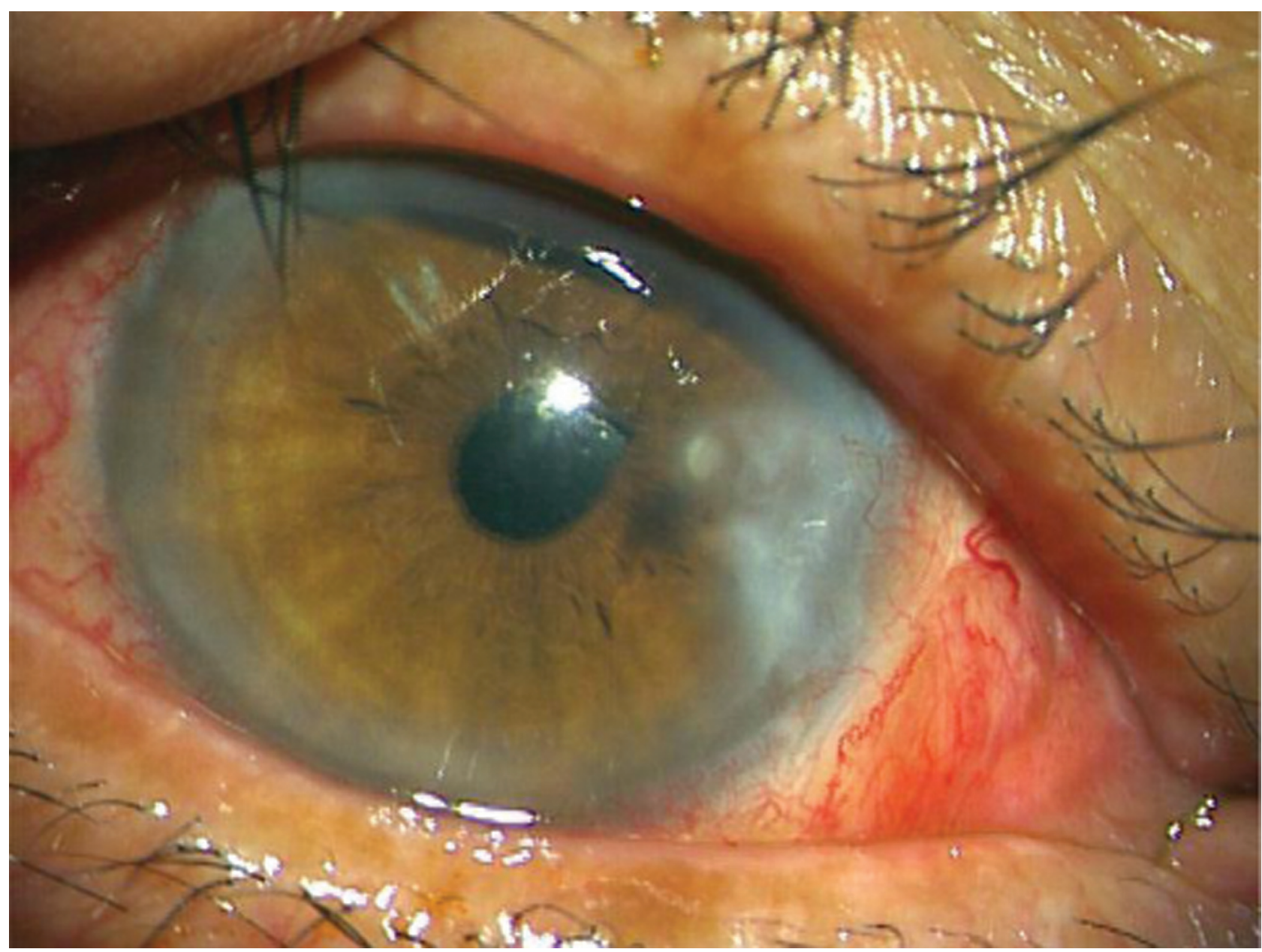

Figure I Clear corneal endothelial plaque was observed at the scar region of the excised pterygium. Note: No remarkable stromal infiltration, no edema, and no epithelial defect.

as a stromal fluffy abcess with stromal infiltration and edema accompanied by endothelial plaque, was observed (Figure 2). Corneal epithelium was eroded and endothelial plaque enlarged with accumulation of hypopyon. For diagnostic purposes, the corneal endothelial plaque was surgically removed with forceps inserted from the limbus, followed by anterior chamber irrigation with calvapenem. The smear of the excised plaque disclosed yeast by Gram staining (Figure 2). Candida albicans was subsequently isolated by culture at Kumamoto City Medical Association Laboratory Center (Kumamoto, Japan). However, samples from aqueous humor and fibrous tissue at angulus iridocornealis were negative in culture. The minimum inhibitory concentration (MIC) of isolated $C$. albicans was fluconazole: $<0.12$, miconazole: $<0.06$, micafungin: $<0.03$, itraconazole: $<0.015 \mu \mathrm{g} / \mathrm{ml}$. $0.2 \%$ fluconazole, $0.2 \%$ micafungin eye drops hourly, and pimaricin ointment once daily with itraconazole (150 mg/day) orally were administered. Three weeks of antimycotic therapy resolved the mycotic keratitis and his visual acuity recovered to 0.7 .

\section{Comments}

The clinical appearance of our patient was modified by steroid administration. Mycotic keratitis is often accompanied by a fluffy abscess with severe stromal edema and infiltration. Epithelial erosion and endothelial plaque with hypopyon are also characteristic of mycotic keratitis. Our patient showed clear endothelial plaque with no epithelial defect, no stromal infiltration, and no stromal edema at his first visit to our hospital as if the pathogenic organism derived intracamerally. Thus we first misdiagnosed it as uveitis or postoperative endophthalmitis. The mycotic organism may possibly have entered the eye during cataract surgery and become attached to the thinned retrocorneal region after pterygium surgery. The rupture of the posterior capsule during cataract surgery also makes diagnosis difficult, and raises the possibility 


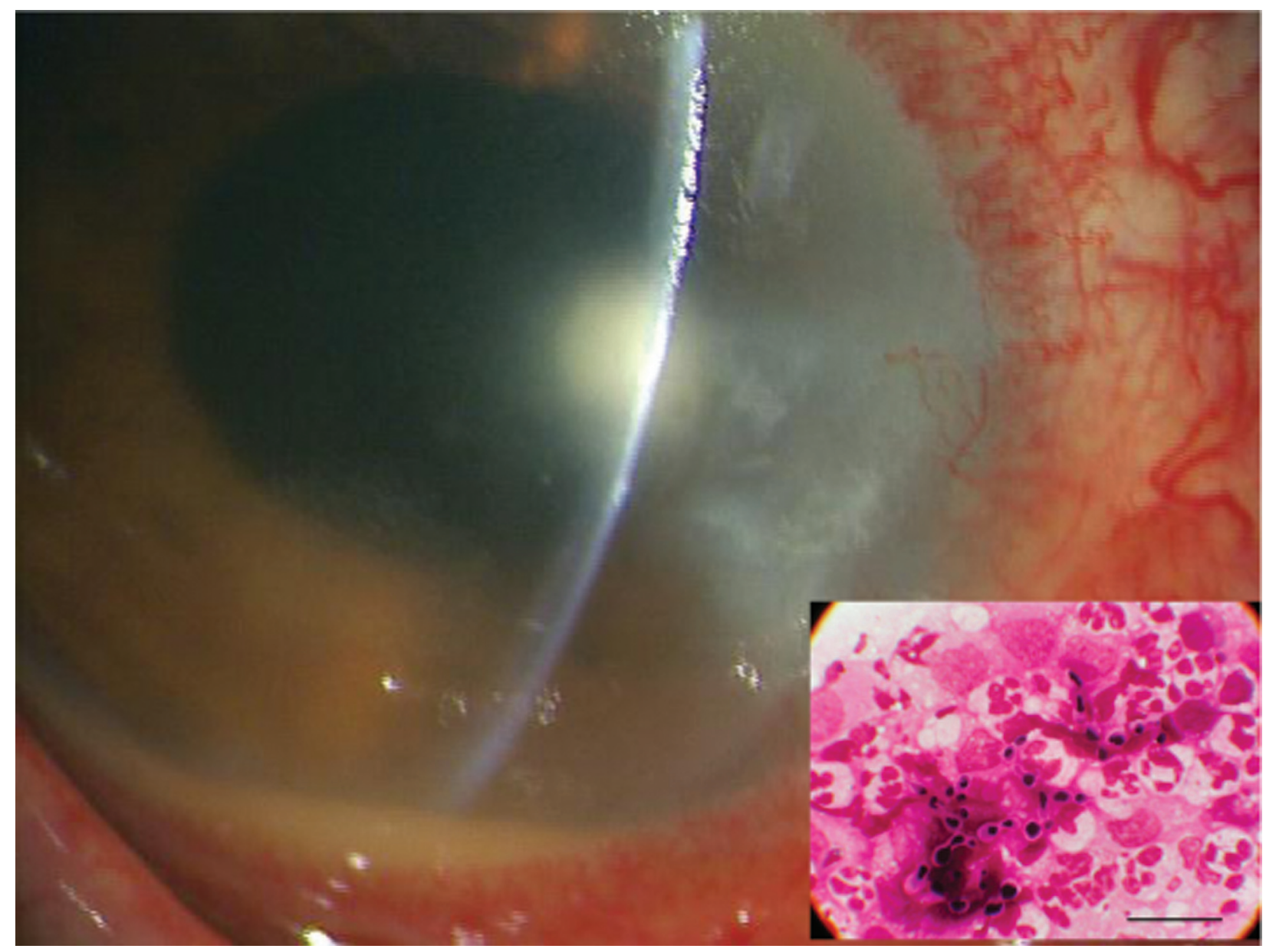

Figure 2 One day after ceasing steroid administration, the corneal endothelial plaque was enlarged and a stromal fluffy abscess with hypopyon formed. Note: Box shows yeast surrounded by many neutrophils and few macrophages, detected by smear of retrocorneal plaque. Bar: $20 \mu \mathrm{m}$.

of endophthalmitis as has been reported..$^{1-3}$ However, we speculate the organism invaded from ocular surface because of the below five reasons. 1) the corneal epithelium was eroded at the patient's first GP visit (Pers comm.); 2) aqueous fluid and fibrous tissue in the anterior chamber included no organism; 3 ) the yeast was surrounded by neutrophils as if leucocytes were preventing the organism spreading into the anterior chamber; 4) the corneal endothelium at the site of the pterygium scar is usually steady-state and may not be the special target of yeast; 5) secession of steroid encouraged mycotic keratitis to progress in the cornea rather than in the intracameral region. For these reasons, we speculate that the pathogenic organism entered through the ocular surface invade immediately through the thinning corneal region at the pterygium scar to reach the endothelium. The inflammation may have been masked by steroid administration and temporary healing of epithelial erosion. Several reports of postoperative infectious keratitis related to a corneal incision such as radial keratotomy, ${ }^{3-7}$ have shown how the characteristics of mycotic keratitis are modified in the thinning region. Aspergilus keratitis and Candida keratitis after radial keratotomy displayed a similar clinical appearance to our case, ${ }^{5,6}$ that is, mycotic focus localized in the deep stromal region accompanied by endothelial plaque with no stromal infiltration, no stromal edema and no epithelial defect as if the organism was derived intracamerally. Thus steroid therapy in the postoperative condition modify the clinical appearance of infectious keratitis. We should pay special attention to prescribing steroid administration for corneal diseases especially in the postoperative condition.

\section{Disclosure}

The authors report no conflicts of interest in this work.

\section{References}

1. Lagnado R, Gupta R, Osborne A, Dua HS. A case of postoperative candida endophthalmitis. Eye. 2005;19:703-705.

2. Narang S, Gupta A, Gupta V, et al. Fungal endophthalmitis following cataract surgery: clinical presentation, microbiological spectrum, and outcome. Am J Ophthalmol. 2001:132:609-617. 
3. Garg P, Mahesh S, Bansal AK, Gopinathan U, Rao GN. Fungal infection of sutureless self-sealing incision for cataract surgery. Ophthalmology. 2003;110:2173-2177.

4. Gussier JR, Miller D, Jaffe M, Alfonso EC. Infection after radial keratotomy. Am J Ophthalmol. 1995;119:798-799.

5. Heidemann DG, Dunn SP, Watts JC. Aspergilus keratitis after radial keratotomy. Am J Ophthalmol. 1995;120:254-256.
6. Maskin SL, Alfonso E. Fungal keratitis after radial keratotomy. Am J Ophthalmol. 1992;114:369-370.

7. Panda A, Das GK, Vanathi M, Kumar A. Corneal infection after radial keratotomy. J Cataract Refract Surg. 1998;24:331-334. 